\section{Through-the-skin ERCP and endoscopic ultrasound-guided cystogastrostomy in a patient with a Roux-en-Y gastric bypass}

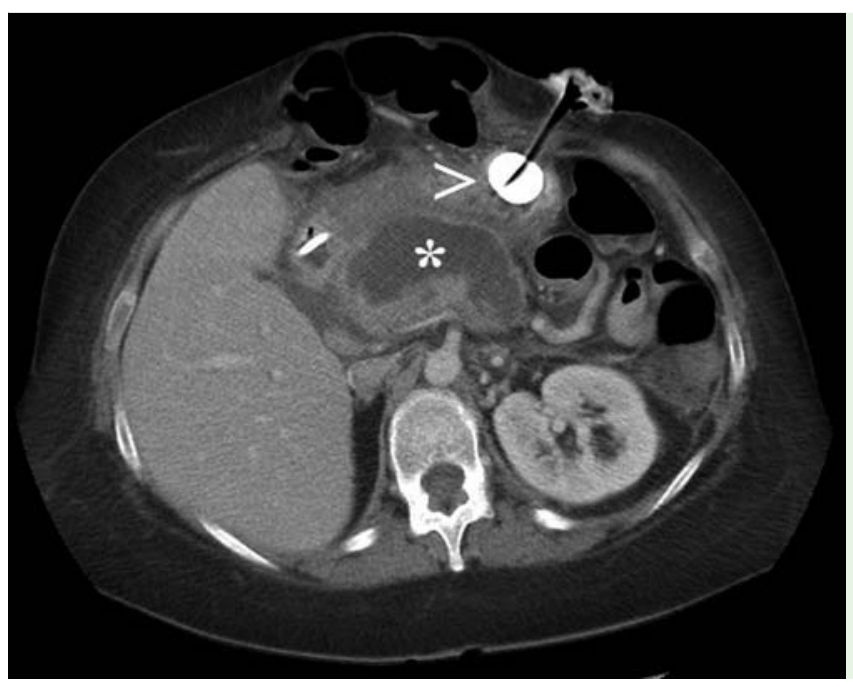

Fig. 1 Computed tomography (CT) scan in a 40-year-old woman with a history of Roux-en-Y gastric bypass showing a fluid collection (white asterisk) adjacent to the excluded stomach which contains the intragastric G-tube balloon (white arrowhead).

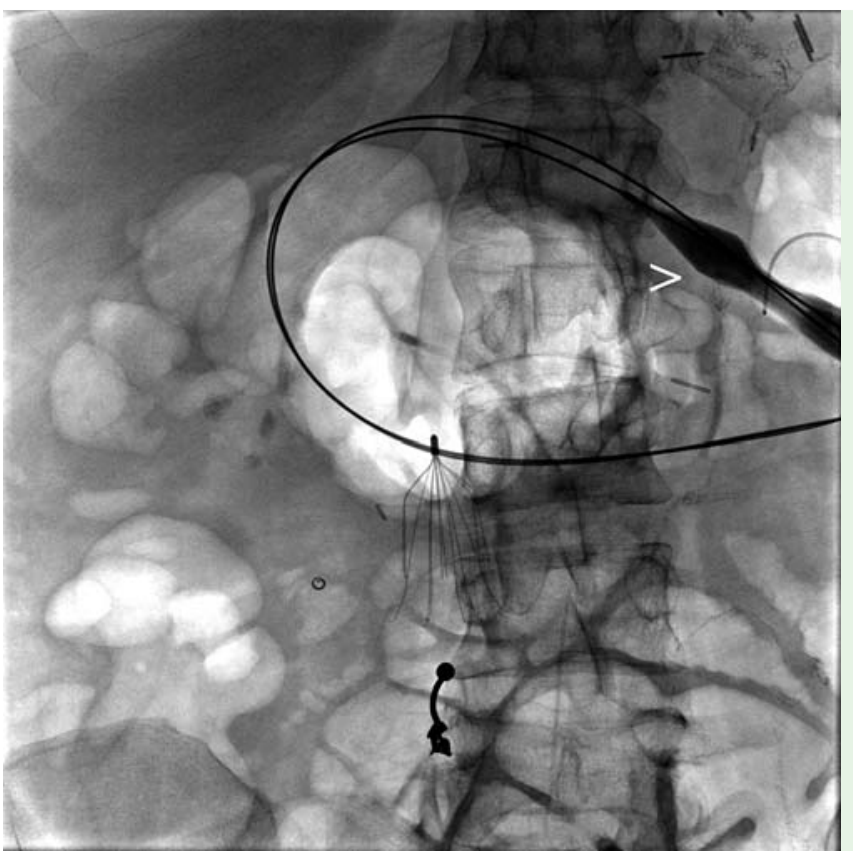

Fig. 2 Fluoroscopic image showing the esophageal dilating balloon (white arrowhead) positioned in the percutaneous tract to the excluded stomach.

A 40-year-old woman with a history of Roux-en-Y gastric bypass (RYGB) 10 years previously was transferred to our institution with acute pancreatitis complicated by acute pancreatic fluid collections (APFCs). The patient complained of severe epigastric abdominal pain and tenderness. Her amylase and lipase, which had peaked at around 5000U/L several days before, were noted to be $116 \mathrm{IU} / \mathrm{L}$ (normal 30-100) and 305U/L (normal 5-50), respectively. Her remaining laboratory re- sults were unremarkable. Computed tomography (CT) and subsequent magnetic resonance imaging (MRI) scans revealed extensive pancreatic stranding and multiple APFCs, including an 8-cm collection that was compressing the duodenum and resulting in distension of the excluded stomach.

Because her symptoms were unremitting and she had outlet obstruction of the gastric remnant, a 22-Fr gastrostomy tube (G-tube) was placed into the excluded stomach under CT guidance to relieve the partial gastric outlet obstruction and allow access to the duodenum for future endoscopic intervention.

She remained symptomatic and a CT scan 6 weeks later confirmed the persistence of a large pseudocyst adjacent to the excluded stomach ( $\bullet$ Fig. 1). After the patient had given informed consent, the G-tube tract was dilated under visual and fluoroscopic guidance to $48 \mathrm{Fr}$ using a standard esophageal dilating balloon ( Fig.2). Once a duodenoscope had been advanced through the tract, pancreatography revealed focal pancreatic duct disruption in the pancreatic head and a stricture in the pancreatic body with upstream dilatation. A 7-Fr, 12-cm pancreatic stent was placed across the disrupted area and the stricture.

The duodenoscope was replaced with a therapeutic linear-array echoendoscope. The cyst was punctured under endoscopic ultrasound (EUS) guidance with a 19-gauge needle. A 0.035-inch guidewire was then coiled within the cyst cavity. The needle tract was dilated with 5,7 , and $10-\mathrm{Fr}$ graduated dilators and then with a $6-\mathrm{mm}$ dilating balloon. A $10-\mathrm{mm}$ fully covered metal stent was placed through this tract across the stomach wall into the lumen of the cyst and a copious amount of fluid was drained. Once the endoscopic procedure had been completed, a 30-Fr G-tube was placed in the tract to allow future endoscopic access to the excluded stomach.

On review 3 weeks later, the patient's abdominal pain had resolved and a CT scan showed complete resolution of the fluid collection during this time. A repeat pancreatogram through the percutaneous tract revealed resolution of the leak and the stricture. The pancreatic and transgastric stents were removed. The G-tube was left in place for an additional 6 weeks in case of pseudocyst recurrence.

Endoscopic retrograde cholangiopancreatography (ERCP) in patients who have undergone RYGB is challenging, but can be performed using enteroscopy-assisted or through-the-skin approaches [1]. Percutaneous access to the excluded stomach, either at the time of laparoscopy or through a mature G-tube tract, allows more reliable identification and cannulation of the papilla and higher therapeutic success rates compared with an enteroscopy-assisted approach [2]. Similarly, percutaneous access to the excluded stomach is necessary for transluminal endoscopic drainage of pancreatic fluid collections 
that are not accessible from the gastric pouch.

In this report, we present a case in which a pancreatic pseudocyst in a patient who had undergone previous RYGB was successfully managed by through-the-skin ERCP and EUS-guided cystogastrostomy. Once access to the excluded stomach had been achieved, we used techniques identical to those employed in patients with non-surgically altered anatomy. Use of a percutaneous approach to achieve pseudocyst drainage has been previously reported [3]; however, our case represents the first report to our knowledge of EUSguided percutaneous pseudocyst drainage. This therapeutic approach should be considered as a novel option for managing pancreatic fluid collections in patients who have undergone RYGB.

Endoscopy_UCTN_Code_TTT_1AS_2AD

Competing interests: None
Joshua Max', William Cooney ${ }^{2}$, James Scheiman ${ }^{3}$, Matthew Dimagno ${ }^{3}$, Badih Elmunzer $^{3}$

${ }^{1}$ Department of Gastroenterology, Cedars-Sinai Medical Center, Los Angeles, California, USA

2 Department of Gastroenterology, The Queen's Medical Center, Honolulu, Hawaii, USA

${ }^{3}$ Department of Gastroenterology, University of Michigan Health System Ann Arbor, Michigan, USA

\section{References}

1 Khashab MA, Okolo PI 3rd. Accessing the pancreatobiliary limb and ERCP in the bariatric patient. Gastrointest Endosc Clin N Am 2011; 21: 305-313

2 Schreiner MA, Chang L, Gluck M et al. Laparoscopy-assisted versus balloon enteroscopy-assisted ERCP in bariatric post-Rouxen-Y gastric bypass patients. Gastrointest Endosc 2012; 75: 748 - 756
3 Tekola B, Wang AY, Ramanath M et al. Percutaneous gastrostomy tube placement to perform transgastrostomy endoscopic retrograde cholangiopancreatography in patients with Roux-en-Y anatomy. Dig Dis Sci 2011; 56: $3364-3369$

Bibliography

Dol http://dx.doi.org/

10.1055/s-0033-1344956

Endoscopy 2014; 46: E22-E23

(c) Georg Thieme Verlag KG

Stuttgart · New York

ISSN 0013-726X

\section{Corresponding author}

Joshua Max, MD

Cedars-Sinai Medical Center - Gastroenterology 8700 Beverly Blvd. South Tower, Suite 7511 Los Angeles

California 90048

USA

Fax: +1-310-423-0146

spinozamax@gmail.com 